\title{
Targeted Metabolomics Approach to Detect the Misuse of Steroidal Aromatase Inhibitors in Equine Sports by Biomarkers Profiling
}

George Ho Man Chan,* Emmie Ngai Man Ho, David Kwan Kon Leung, Kin Sing Wong and Terence See Ming Wan*

Racing Laboratory, The Hong Kong Jockey Club, Sha Tin Racecourse, Sha Tin, N.T., Hong Kong, China

Supporting Information 


\section{Table of Contents}

\section{Page S-3 Experimental}

Page S-6 Table S1 GC-MS/MS experimental parameters of target steroids and their corresponding internal standards (IS).

Page S-7 Table S2 Inter-day precision, accuracy, extraction recovery of spiked quality control samples.

Page S-8 Table S3 Intra-day precision, limit of detection (LoD) and limit of quantification (LoQ) of spiked quality control samples $(n=6)$.

Page S-9 Figure S1 Three dimensional PCA loading plot with respect to the treatment classification and post administration time profile.

Page S-10 Figure S2 Validation plot obtained from 100 permutation tests of the OPLS-DA model.

Page S-11 Figure S3 Urinary elimination profiles of (A) testosterone; (B) $5 \alpha$-androstane$3 \beta, 17 \alpha$-diol; (C) $5 \alpha$-androstane-3 $\beta, 17 \beta$-diol; (D) androsterone; (E) 5-androstene-

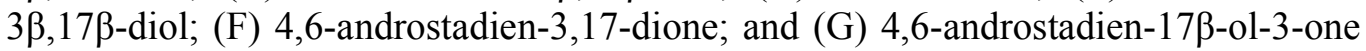
following oral treatment with respectively 6-OXO (2 geldings, Horses 1 and 2, blue and red lines) and ATD (2 geldings, Horses 3 and 4, green and purple lines).

\section{Page S-12 References}




\section{EXPERIMENTAL}

\section{Materials}

Progesterone and nandrolone were obtained from British Pharmacopoeia (Middlesex, UK). 19Norepiandrosterone, estrone and androsterone were purchased from Alltech (Derrfield, IL, USA). 17 $\beta$-estradiol was obtained from United States Pharmacopeia (Rockville, MD, USA). Testosterone

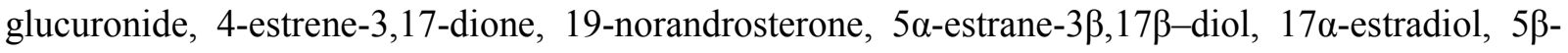

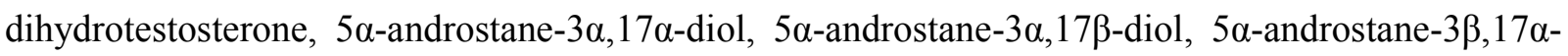
diol, 19-noretiocholanolone, androstanedione, etiocholanedione, 5-androstene-3 $\beta, 17 \beta$-diol, boldione, 4,6-androstadien-3,17-dione and 4,6-androstadien-17ß-ol-3-one were obtained from Steraloids (Newport, RI, USA). Testosterone, $\beta$-estradiol-3-( $\beta$-D-glucuronide)-17-sulfate, $\beta$-estradiol-3-

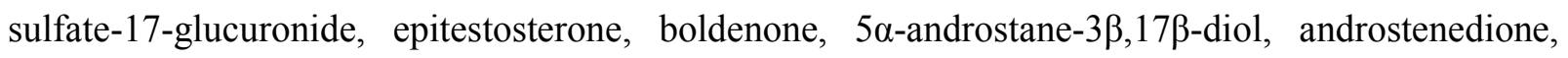
epiandrosterone, dehydroepiandrosterone, and stanolone were purchased from Sigma-Aldrich (St.

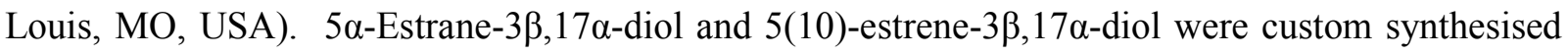
by the Hong Kong University of Science and Technology. ${ }^{1}$ Testosterone sulfate, $d_{3}$-testosterone sulfate and $d_{3}$-nandrolone were obtained from National Measurement Institute (Sydney, Australia). $d_{3}$-Androstane-3 $\alpha, 17 \beta$-diol ( $d_{3}$-androstanediol) was synthesised and characterised in-house.

Chloroform (GR grade), diisopropyl ether, $n$-hexane (GR grade), $n$-heptane (LC grade), ammonium sulfate $(\geq 99.5 \%$ ), sodium chloride $(\geq 99.5 \%$ ) and methanol (LiChrosolv grade) were obtained from Merck (Darmstadt, Germany). Ethyl acetate (GR grade), acetonitrile (LC Grade), sodium hydroxide $(\geq 98 \%)$ were obtained from Sigma-Aldrich. Pentafluoropropionic acid anhydride (PFPA) was obtained from Pierce (Rockford, IL, USA). $\quad \beta$-Glucuronidase from E. Coli. K12 was obtained from Roche (Indianapolis, IN, USA). Anhydrous methanolic hydrogen chloride used for methanolysis was prepared according to the procedures reported previously. ${ }^{2}$ Deionised water was generated from an in-house water purification system (Milli-Q, Mosheim, France). Sep-Pak Vac C18 solid phase 
extraction (SPE) cartridge (3 mL, $500 \mathrm{mg}$ ) was supplied by Waters Corporation (Milford, MA, USA). ABS Elut NEXUS SPE cartridge ( $3 \mathrm{~mL}, 60 \mathrm{mg}$ ) was supplied by Agilent Technologies (Santa Clara, CA, USA).

\section{In-house drug administration and control samples}

Control (untreated) urine samples were collected from twenty-four healthy castrated male (gelding) thoroughbreds (imported from different countries in both hemispheres and aged between 3 and 9 years old) over a period from December 2013 to April 2014. Sampling was conducted under prerace conditions on the morning of a raceday.

The administration protocols have already been published by the authors' laboratory. ${ }^{3,4}$ Briefly, a dose of $500 \mathrm{mg}$ of androst-4-ene-3,6,17-tione (6-OXO) was administered orally to each of two thoroughbred geldings (aged 7 at $0.97 \mathrm{mg} / \mathrm{kg}$ and 11 at $0.98 \mathrm{mg} / \mathrm{kg}$ ) by stomach tubing. Pre- and post-administration urine samples were collected at least twice daily for up to 6 days post administration. ${ }^{3}$ For ATD, a dose of $800 \mathrm{mg}$ of androsta-1,4,6-triene-3,17-dione (ATD) was administered orally to each of two thoroughbred geldings (aged 7 at $1.58 \mathrm{mg} / \mathrm{kg}$ and 8 at $1.63 \mathrm{mg} / \mathrm{kg}$ ) by stomach tubing. Pre- and post-administration urine samples were collected at least twice daily for the first three days, and then once every day up to 9 days. ${ }^{4}$ In this study, pre-administration urine samples were taken as untreated samples and were included in the control group. All samples were aliquoted as soon as possible upon receipt, and the aliquots were stored at around $-80^{\circ} \mathrm{C}$ before analysis. Aliquots of administration urine samples as collected in the previous study were retrieved and analysed in this study. The administration experiments were approved in accordance with the Animals (Control of Experiments) Ordinance by the Licensing Authority of the Department of Health, the Government of the Hong Kong Special Administrative Region. 


\section{Deconjugation study}

The efficiency of the deconjugation procedure was evaluated using testosterone sulfate (TS), testosterone glucuronide (TG), $\beta$-estradiol-3-( $\beta$-D-glucuronide)-17-sulfate (E2-3G17S) and $\beta$ estradiol-3-sulfate-17-glucuronide (E2-3S17G) as model compounds. Each of the conjugated steroids (equivalent to $50 \mathrm{ng} / \mathrm{mL}$ of free steroid) was spiked into a blank horse urine sample in duplicate and processed using the method described above. The concentrations of the deconjugated steroids and recoveries were estimated using a calibrator spiked with a standard mixture of testosterone $(50 \mathrm{ng} / \mathrm{mL})$ and $17 \beta$-estradiol $(50 \mathrm{ng} / \mathrm{mL})$ in blank urine analysed in parallel.

It is well known that many AAS undergo extensive phase II metabolism in the mammalian body. ${ }^{5-7}$ In this study, urine samples were subjected to both enzyme hydrolysis and methanolysis in order to maximise the recoveries of free steroids from their glucuronide and/or sulfate conjugates, and hence provide a better estimate of the total amount of target steroids in the samples. The deconjugation efficiency of the method was evaluated using TS, TG, E2-3G17S, and E2-3S17G as model compounds. Our results showed that both TS and TG had very high recoveries at $126 \%$ and $106 \%$ respectively whereas the mixed conjugates E2-3G17S and E2-3S17G were lower at $76 \%$ and $78 \%$ respectively. Herein, the recoveries of TS and TG were found to be greater than $100 \%$, which could be attributed to the slight difference in extraction efficiency between free testosterone in the calibrator and conjugated testosterone in the hydrolysed spiked urine samples. On the other hand, the lower recoveries of the mixed conjugates steroids could be attributed to their possible loss during the SPE steps, or else the deconjugation steps used might be less effective on mixed conjugates. However, no attempt was made to verify the possible causes. 
Table S1 GC-MS/MS experimental parameters of target steroids and their corresponding internal standards (IS).

\begin{tabular}{|c|c|c|c|c|c|c|c|}
\hline Analyte & Abbr. & $\begin{array}{c}\text { RT } \\
(\mathrm{min})\end{array}$ & $\begin{array}{c}\text { Quantitative } \\
\text { transition }\end{array}$ & $\begin{array}{c}\mathrm{CE} \\
(\mathrm{eV})\end{array}$ & $\begin{array}{c}\text { Qualitative } \\
\text { transition } \\
\end{array}$ & $\begin{array}{c}\mathrm{CE} \\
(\mathrm{eV})\end{array}$ & IS \\
\hline 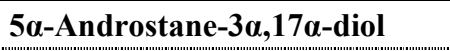 & $\alpha \alpha \alpha$ & 8.54 & $420.1>148.2$ & 8 & $420.1>149.2$ & 12 & $d_{3}-\mathrm{A}$ \\
\hline 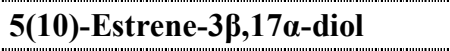 & EED & 8.60 & $404.1>225.2$ & 14 & $404.1>240.2$ & 6 & $d_{3}-\mathrm{A}$ \\
\hline 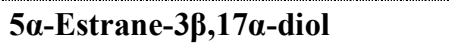 & $\operatorname{EAD}(\alpha \beta \alpha)$ & 8.82 & $406.1>391.2$ & 6 & $406.1>242.2$ & 6 & $d_{3}-\mathrm{A}$ \\
\hline$d_{3}$-Androstanediol & $d_{3}-\mathrm{A}$ & 9.14 & $423.2>244.2$ & 12 & \multicolumn{3}{|c|}{ Internal Standard } \\
\hline 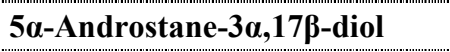 & $\alpha \alpha \beta$ & 9.15 & $420.2>148.2$ & 6 & $420.2>149.2$ & 12 & $d_{3}-\mathrm{A}$ \\
\hline 19-Norandrosterone & 19-NA & 9.16 & $422.1>378.2$ & 6 & $422.1>199.2$ & 18 & $d_{3}-\mathrm{A}$ \\
\hline Boldenone & $\mathrm{B}$ & 9.17 & $578.1>414.1$ & 6 & $578.1>251.2$ & 14 & $d_{3}-\mathrm{T}$ \\
\hline Epitestosterone & $\mathrm{E}$ & 9.18 & $580.1>253.2$ & 10 & $580.1>146.2$ & 8 & $d_{3}-T$ \\
\hline 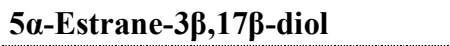 & $\operatorname{EAD}(\alpha \beta \beta)$ & 9.20 & $406.1>201.2$ & 8 & $406.1>159.1$ & 12 & $d_{3}-\mathrm{A}$ \\
\hline 17a-Estradiol & $\mathrm{E} 2 \alpha$ & 9.22 & $564.1>237.2$ & 10 & $564.1>400.1$ & 6 & $d_{3}-\mathrm{T}$ \\
\hline 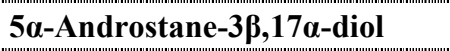 & $\alpha \beta \alpha$ & 9.27 & $420.1>148.2$ & 8 & $420.1>149.2$ & 12 & $d_{3}-\mathrm{A}$ \\
\hline$d_{3}$-Nandrolone & $d_{3}-\mathrm{N}$ & 9.36 & $569.1>148.1$ & 10 & \multicolumn{3}{|c|}{ Internal Standard } \\
\hline Nandrolone & $19-\mathrm{NT}$ & 9.38 & $566.1>133.1$ & 16 & $566.1>146.2$ & 8 & $d_{3}-\mathrm{N}$ \\
\hline 4,6-Androstadien-17 $\beta$-ol-3-one & $6-\mathrm{T}$ & 9.39 & $578.1>133.2$ & 16 & $578.1>280.1$ & 8 & $d_{3}-\mathrm{T}$ \\
\hline 19-Noretiocholanolone & 19-NE & 9.43 & $422.1>378.1$ & 6 & $422.1>199.2$ & 20 & $d_{3}-\mathrm{A}$ \\
\hline$d_{3}$-Testosterone & $d_{3}-T$ & 9.51 & $583.1>568.2$ & 10 & \multicolumn{3}{|c|}{ Internal Standard } \\
\hline Testosterone & $\mathrm{T}$ & 9.52 & $580.1>270.1$ & 8 & $580.1>146.2$ & 10 & $d_{3}-\mathrm{T}$ \\
\hline 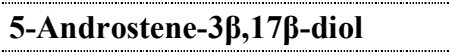 & AED & 9.54 & $418.1>183.1$ & 10 & $418.1>121.1$ & 10 & $d_{3}-\mathrm{A}$ \\
\hline 17 $\beta$-Estradiol & E2 & 9.57 & $564.1>237.2$ & 8 & $564.1>401.2$ & 6 & $d_{3}-\mathrm{T}$ \\
\hline Androsterone & A & 9.61 & $436.1>392.1$ & 6 & $436.1>213.2$ & 22 & $d_{3}-\mathrm{A}$ \\
\hline 19-Norepiandrosterone & 19-NEA & 9.63 & $422.1>378.1$ & 6 & $422.1>199.2$ & 22 & $d_{3}-\mathrm{A}$ \\
\hline 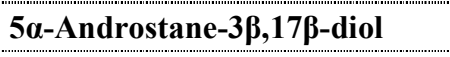 & $\alpha \beta \beta$ & 9.67 & $420.2>148.2$ & 6 & $420.2>149.2$ & 10 & $d_{3}-A$ \\
\hline 4-Estrene-3,17-dione & NOR & 9.80 & $418.1>149.1$ & 12 & $418.1>105.1$ & 26 & $\begin{array}{ll}d_{3}-T \\
d_{3}-T\end{array}$ \\
\hline 4,6-Androstadien-3,17-dione & 6-ADD & 9.83 & $430.1>135.1$ & 10 & $430.1>107.1$ & 30 & $d_{3}-\mathrm{T}$ \\
\hline Boldione & 1-ADD & 9.85 & $430.1>264.1$ & 8 & $430.1>235.1$ & 20 & $d_{3}-\mathrm{T}$ \\
\hline Androstenedione & ADIONE & 9.98 & $432.1>149.1$ & 12 & $432.1>417.1$ & 8 & $d_{3}-T$ \\
\hline Estrone & E1 & 9.99 & $416.1>372.1$ & 8 & $416.1>253.2$ & 6 & $d_{3}-\mathrm{T}$ \\
\hline Dehydroepiandrosterone & DHEA & 10.00 & $270.1>121.1$ & 10 & $270.1>199.1$ & 6 & $d_{3}-\mathrm{A}$ \\
\hline 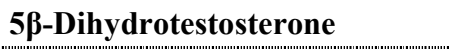 & $5 \beta-\mathrm{DHT}$ & 10.08 & $436.1>366.2$ & 8 & $436.1>201.2$ & 16 & $d_{3}-\mathrm{A}$ \\
\hline Epiandrosterone & EA & 10.15 & $436.2>213.2$ & 22 & $436.2>239.2$ & 16 & $d_{3}-\mathrm{A}$ \\
\hline Stanolone & $5 \alpha-\mathrm{DHT}$ & 10.33 & $436.2>364.1$ & 8 & $436.2>201.2$ & 16 & $d_{3}-\mathrm{A}$ \\
\hline Etiocholanedione & $5 \beta$-DIONE & 10.60 & $288.2>244.2$ & 6 & $288.2>255.2$ & 8 & $d_{3}-\mathrm{A}$ \\
\hline Androstanedione & $5 \alpha$-DIONE & 10.86 & $288.2>244.2$ & 6 & $288.2>255.2$ & 8 & $d_{3}-\mathrm{A}$ \\
\hline Progesterone & $\mathrm{P} 4$ & 10.88 & $460.1>147.1$ & 10 & $460.1>455.2$ & 8 & $d_{3}-\mathrm{T}$ \\
\hline
\end{tabular}


Table S2 Inter-day precision, accuracy, extraction recovery of spiked quality control samples.

\begin{tabular}{|c|c|c|c|c|c|c|c|}
\hline \multirow[b]{2}{*}{ Analyte } & \multicolumn{2}{|c|}{$5 \mathrm{ng} / \mathrm{mL}$} & \multicolumn{2}{|c|}{$20 \mathrm{ng} / \mathrm{mL}$} & \multicolumn{2}{|c|}{$40 \mathrm{ng} / \mathrm{mL}$} & \multirow{2}{*}{$\begin{array}{c}\text { Extraction recovery } \\
(\%)\end{array}$} \\
\hline & $\begin{array}{c}\text { Accuracy } \\
(\%) \\
\end{array}$ & $\begin{array}{c}\begin{array}{c}\text { Precision } \\
(\% \mathrm{RSD})\end{array} \\
\end{array}$ & $\begin{array}{c}\text { Accuracy } \\
(\%)\end{array}$ & $\begin{array}{c}\begin{array}{c}\text { Precision } \\
(\% \text { RSD })\end{array} \\
\end{array}$ & $\begin{array}{c}\text { Accuracy } \\
(\%) \\
\end{array}$ & $\begin{array}{c}\begin{array}{c}\text { Precision } \\
(\% \text { RSD) }\end{array} \\
\end{array}$ & \\
\hline$\alpha \alpha \alpha$ & 101 & 7.4 & 106 & 8.7 & 114 & 8.8 & 45.4 \\
\hline EED & 94 & 2.8 & 100 & 6.8 & 100 & 6.9 & 43.6 \\
\hline EAD $(\alpha \beta \alpha)$ & 96 & 7.3 & 101 & 4.4 & 104 & 4.1 & 64.2 \\
\hline$\alpha \alpha \beta$ & 91 & 5.7 & 96 & 3.5 & 102 & 6.2 & 51.5 \\
\hline 19-NA & 90 & 2.6 & 97 & 4.3 & 99 & 5.0 & 51.7 \\
\hline B & 110 & 6.7 & 111 & 7.7 & 107 & 7.0 & 65.0 \\
\hline $\mathbf{E}$ & 106 & 4.7 & 108 & 6.1 & 106 & 4.5 & 62.6 \\
\hline EAD $(\alpha \beta \beta)$ & 87 & 5.9 & 93 & 6.9 & 95 & 4.7 & 59.0 \\
\hline $\mathbf{E} 2 \alpha$ & 108 & 11.1 & 108 & 10.2 & 109 & 14.1 & 19.8 \\
\hline$\alpha \beta \alpha$ & 97 & 2.9 & 100 & 4.5 & 104 & 3.0 & 53.3 \\
\hline 19-NT & 97 & 4.2 & 102 & 5.7 & 103 & 6.0 & 61.2 \\
\hline $6-T$ & 108 & 30.7 & 91 & 34.9 & 93 & 43.2 & 59.2 \\
\hline 19-NE & 93 & 2.8 & 98 & 2.6 & 103 & 2.5 & 60.4 \\
\hline T & 105 & 4.2 & 105 & 5.7 & 103 & 1.6 & 62.7 \\
\hline AED & 94 & 5.9 & 99 & 7.7 & 101 & 5.2 & 62.4 \\
\hline E2 & 104 & 12.3 & 103 & 9.5 & 101 & 11.9 & 17.6 \\
\hline A & 95 & 5.8 & 97 & 4.9 & 101 & 4.2 & 47.5 \\
\hline 19-NEA & 92 & 6.2 & 99 & 5.4 & 101 & 2.9 & 55.3 \\
\hline$\alpha \beta \beta$ & 93 & 4.5 & 96 & 6.5 & 99 & 2.7 & 56.7 \\
\hline NOR & 99 & 6.9 & 101 & 8.6 & 97 & 2.7 & 58.2 \\
\hline 6-ADD & 105 & 18.5 & 90 & 21.6 & 91 & 25.5 & 58.1 \\
\hline 1-ADD & 100 & 8.1 & 101 & 8.0 & 99 & 6.0 & 64.1 \\
\hline ADIONE & 102 & 5.4 & 103 & 8.7 & 99 & 3.5 & 60.6 \\
\hline E1 & 92 & 12.1 & 97 & 11.2 & 97 & 13.7 & 17.4 \\
\hline DHEA & 91 & 5.5 & 96 & 8.4 & 98 & 7.3 & 57.0 \\
\hline $5 \beta$-DHT & 101 & 6.9 & 110 & 6.6 & 115 & 9.7 & 54.0 \\
\hline EA & 99 & 8.6 & 100 & 9.3 & 95 & 12.9 & 51.1 \\
\hline $5 \alpha-\mathrm{DHT}$ & 113 & 10.7 & 125 & 11.9 & 128 & 12.4 & 49.6 \\
\hline 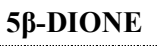 & 105 & 14.4 & 110 & 11.1 & 115 & 11.3 & 52.4 \\
\hline 5a-DIONE & 109 & 12.3 & 119 & 11.3 & 120 & 10.3 & 49.2 \\
\hline P4 & 96 & 6.2 & 99 & 13.1 & 97 & 5.8 & 30.9 \\
\hline
\end{tabular}


Table S3 Intra-day precision, limit of detection (LoD) and limit of quantification (LoQ) of spiked quality control samples $(n=6)$.

\begin{tabular}{|c|c|c|c|}
\hline Analyte & $\begin{array}{c}5 \mathrm{ng} / \mathrm{mL} \\
\begin{array}{c}\text { Intra-day precision } \\
(\% \mathrm{RSD})\end{array} \\
\end{array}$ & $\begin{array}{c}\text { LoD } \\
(\mathrm{ng} / \mathrm{mL})\end{array}$ & $\begin{array}{c}\text { LoQ } \\
(\mathrm{ng} / \mathrm{mL})\end{array}$ \\
\hline$\alpha \alpha \alpha$ & 3.3 & 0.5 & 1.7 \\
\hline EED & 7.6 & 1.1 & 3.5 \\
\hline $\operatorname{EAD}(\boldsymbol{\alpha} \boldsymbol{\beta} \alpha)$ & 2.5 & 0.4 & 1.2 \\
\hline$\alpha \alpha \beta$ & 2.7 & 0.4 & 1.2 \\
\hline 19-NA & 4.0 & 0.6 & 1.9 \\
\hline B & 2.9 & 0.5 & 1.7 \\
\hline $\mathbf{E}$ & 4.8 & 0.9 & 2.9 \\
\hline EAD $(\boldsymbol{\alpha \beta \beta})$ & 2.8 & 0.4 & 1.2 \\
\hline $\mathrm{E} 2 \alpha$ & 2.3 & 0.4 & 1.4 \\
\hline$\alpha \beta \alpha$ & 2.1 & 0.3 & 1.1 \\
\hline 19-NT & 2.1 & 0.3 & 1.1 \\
\hline $6-T$ & 11.4 & 1.9 & 6.2 \\
\hline 19-NE & 4.0 & 0.5 & 1.8 \\
\hline $\mathbf{T}$ & 3.3 & 0.5 & 1.8 \\
\hline AED & 2.0 & 0.3 & 0.9 \\
\hline E2 & 2.5 & 0.4 & 1.4 \\
\hline A & 4.6 & 0.6 & 1.8 \\
\hline 19-NEA & 4.4 & 0.6 & 2.0 \\
\hline$\alpha \beta \beta$ & 3.1 & 0.4 & 1.4 \\
\hline NOR & 3.8 & 0.6 & 1.9 \\
\hline 6-ADD & 5.6 & 1.0 & 3.2 \\
\hline 1-ADD & 2.2 & 0.4 & 1.2 \\
\hline ADIONE & 2.2 & 0.3 & 1.0 \\
\hline E1 & 2.6 & 0.4 & 1.4 \\
\hline DHEA & 2.6 & 0.4 & 1.3 \\
\hline $5 \beta$-DHT & 5.6 & 0.7 & 2.5 \\
\hline EA & 5.1 & 0.8 & 2.8 \\
\hline $5 \alpha-D H T$ & 5.1 & 0.7 & 2.4 \\
\hline 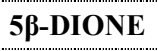 & 4.3 & 0.6 & 2.0 \\
\hline 5a-DIONE & 4.4 & 0.6 & 2.1 \\
\hline P4 & 6.9 & 1.2 & 4.1 \\
\hline
\end{tabular}




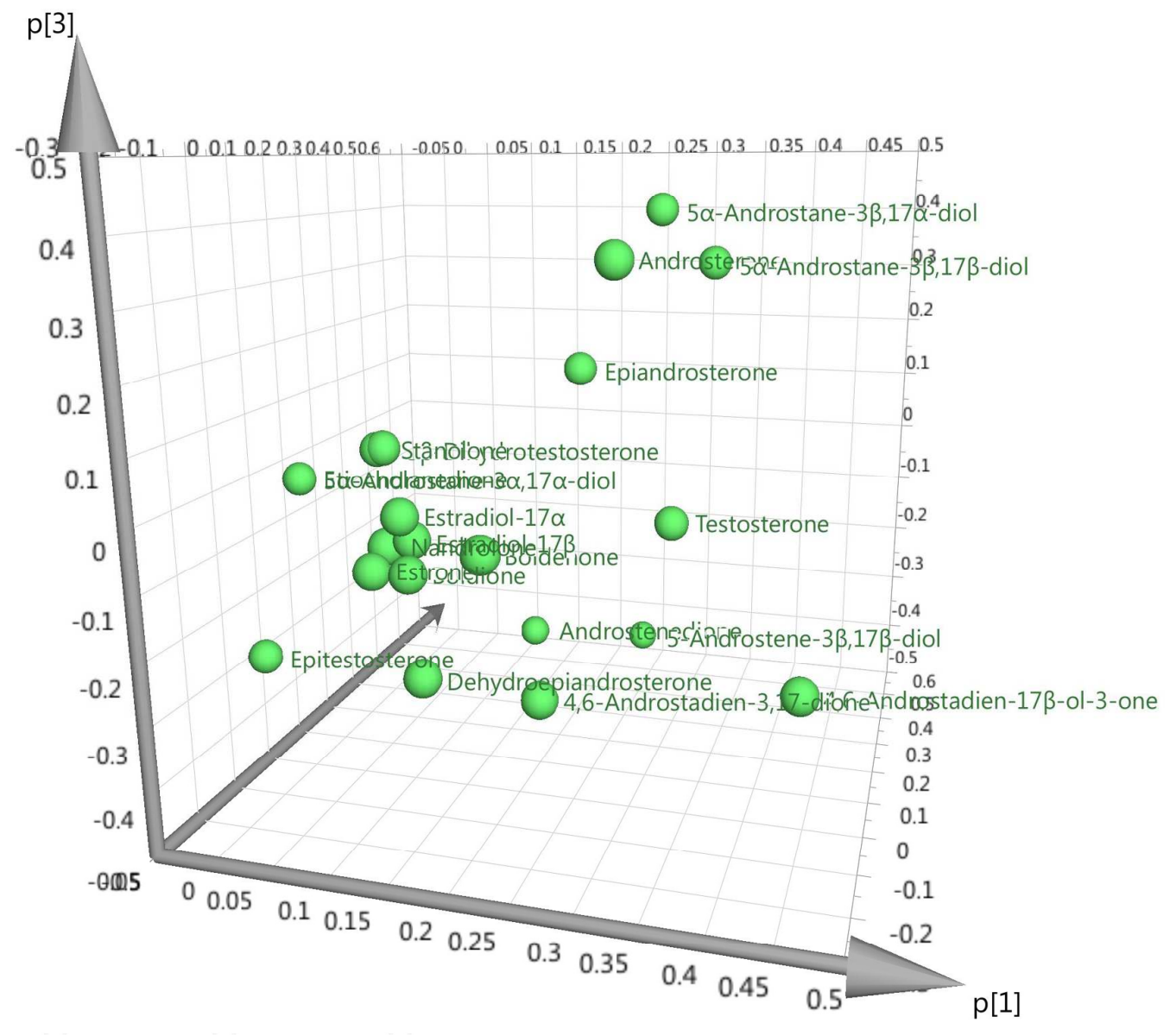

Figure S1 Three dimensional PCA loading plot with respect to the treatment classification and post administration time profile. 


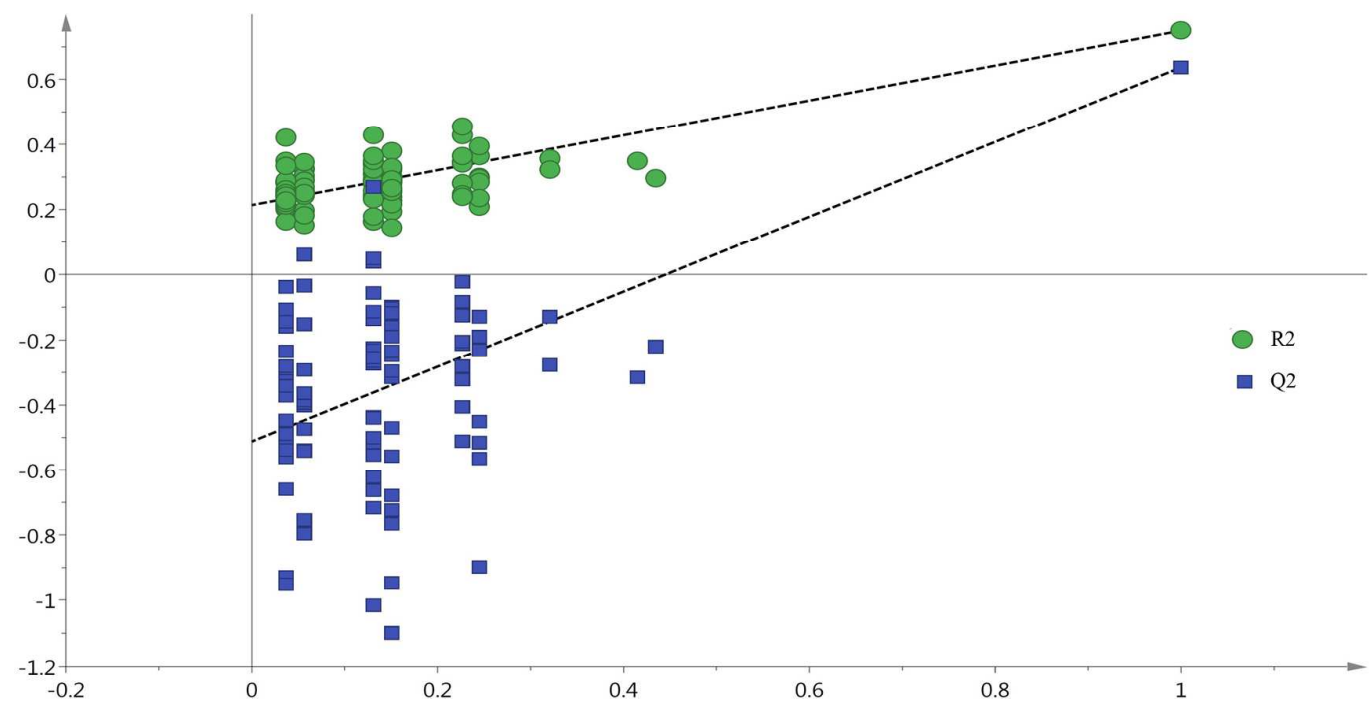

Figure S2 Validation plot obtained from 100 permutation tests of the OPLS-DA model. 

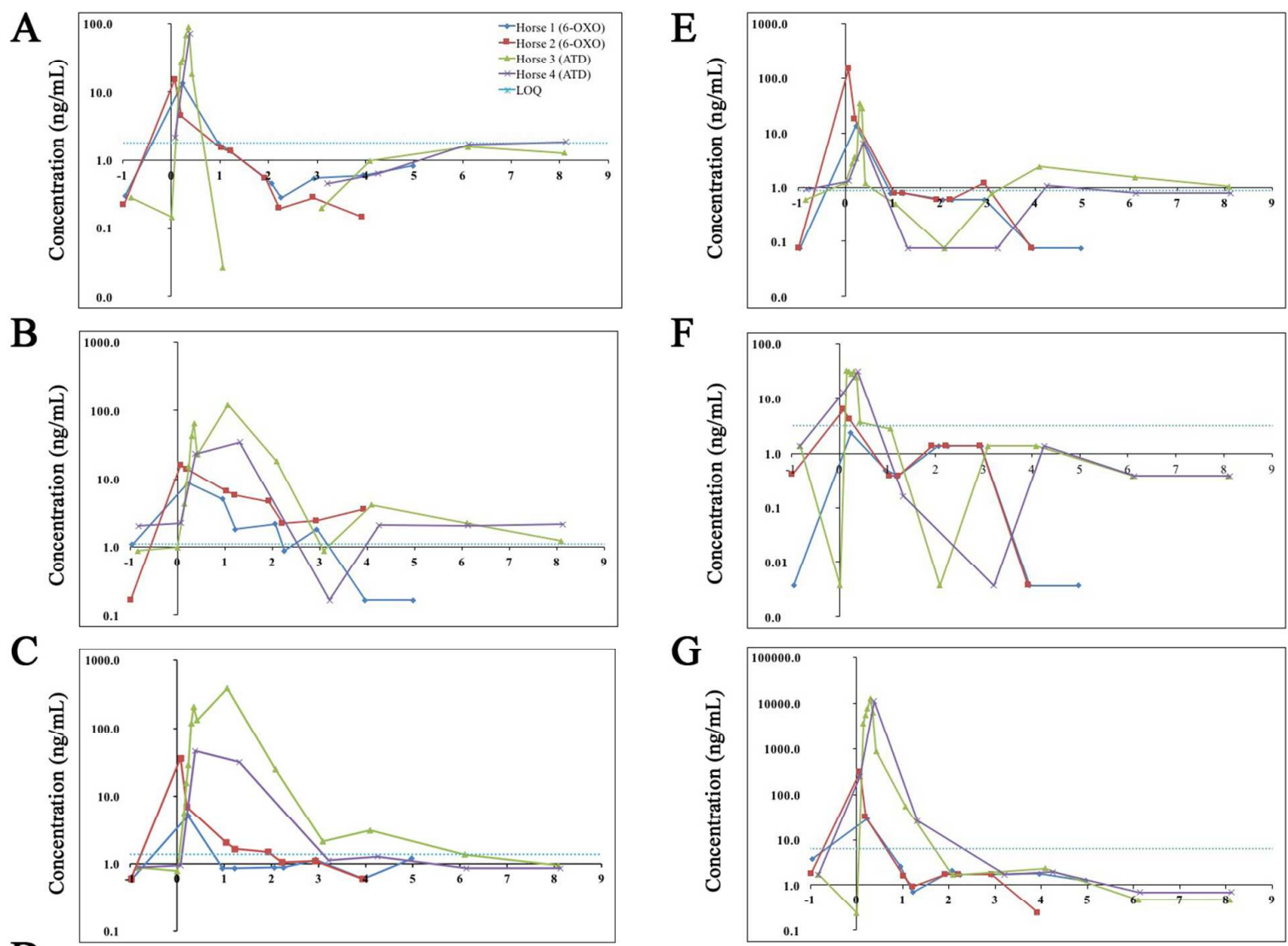

$\mathrm{D}$

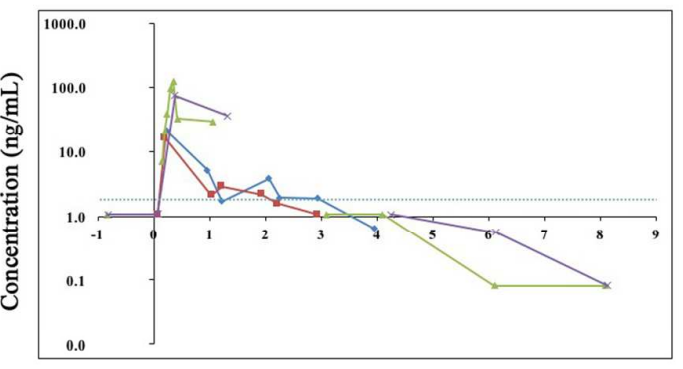

Post-administration Days

Post-administration Days

Figure S3 Urinary elimination profiles of (A) testosterone; (B) $5 \alpha$-androstane-3 $\beta, 17 \alpha$-diol; (C) $5 \alpha$ -

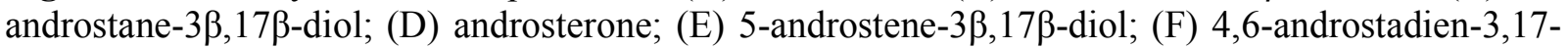
dione; and $(\mathrm{G})$ 4,6-androstadien-17 $\beta$-ol-3-one following oral treatment with respectively 6-OXO (2 geldings, Horses 1 and 2, blue and red lines) and ATD ( 2 geldings, Horses 3 and 4, green and purple lines). 


\section{References:}

(1) Tang, P. W.; Crone, D. L.; Chan, A. W. O.; Hui, K. N.; Williams, I. D.; Wan, T. S. M. Proc. 10th Int. Conf. Racing Anal. Vet., Stockholm 1995; R\&W Publications: Newmarket; 329.

(2) Tang, P. W.; Crone, D. L. Anal Biochem 1989, 182, 289-294.

(3) Leung, G. N. W.; Tang, F. P. W.; Wan, T. S. M.; Wong, C. H. F.; Lam, K. K.; Stewart, B. D. Biomed Chromatogr 2010, 24, 744-751.

(4) Kwok, W. H.; Leung, G. N. W.; Wan, T. S. M.; Curl, P.; Schiff, P. J. J Steroid Biochem Mol Biol 2015, 152, 142-154.

(5) Dumasia, M. C.; Houghton, E. Xenobiotica 1981, 11, 323-331.

(6) Dumasia, M. C.; Houghton, E. Xenobiotica 1984, 14, 647-655.

(7) Teale, P.; Houghton, E. Bioanalysis 2010, 2, 1085-1107. 